\title{
QuantPipe: A user-friendly pipeline software tool for DIA data analysis based on the OpenSWATH-PyProphet-TRIC workflow
}

\author{
Dazheng Wang ${ }^{1}$, Guohong Gan ${ }^{1}$, Xi Chen ${ }^{2,3}$, Chuan-Qi Zhong ${ }^{1 *}$
}

${ }^{1}$ State Key Laboratory of Cellular Stress Biology, Innovation Center for Cellular Signaling Network, School of Life

Sciences, Xiamen University, China.

${ }^{2}$ SpecAlly Life Technology Co., Ltd, Wuhan, China.

${ }^{3}$ Medical Research Institute, Wuhan University, Wuhan, China.

*Correspondence should be addressed to C.-Q. Z (zhongcq@xmu.edu.cn).

Figure S1A. Screenshot of OpenSWATH-PyProphet-TRIC workflow parameters setting.

Figure S1B. Screenshot of QuantPipe's parameters setting.

Figure S1C. Overlap of identified proteins by OpenSWATH (QuantPipe), directDIA Spectronaut and library-based Spectronaut.

Figure S1D.Screenshot of QuantPipe. The checkbox indicated by the red arrow is enabling ion mobility that was used to peak scoring in mobi-DIK through the QuantPipe interface.

Figure S1E. The number of peptides in the spectral library from 12 phospho-DDA runs.

Figure S1F. Screenshot of QuantPipe. The checkbox indicated by the red arrow is enabling IPF that was used to analyze PTM-specific peptide through the QuantPipe interface.

Table S1. Identified and quantified peptides and proteins in TripleTOF 5600 SWATH-MS data.

Table S2. Identified and quantified peptides and proteins in TripleTOF 6600 SWATH-MS data.

Table S3. Identified and quantified peptides and proteins in Orbitrap fusion lumos DIA data.

Table S4. Identified and quantified peptides and proteins in timsTOF Pro diaPASEF data.

Table S5. Identified and quantified peptides in TripleTOF 5600 phospho-SWATH-MS data.

Table S6. Identified and quantified peptides and proteins in large-scale of TripleTOF 5600 SWATHMS data. 


\section{Supplementary Figure 1}

A

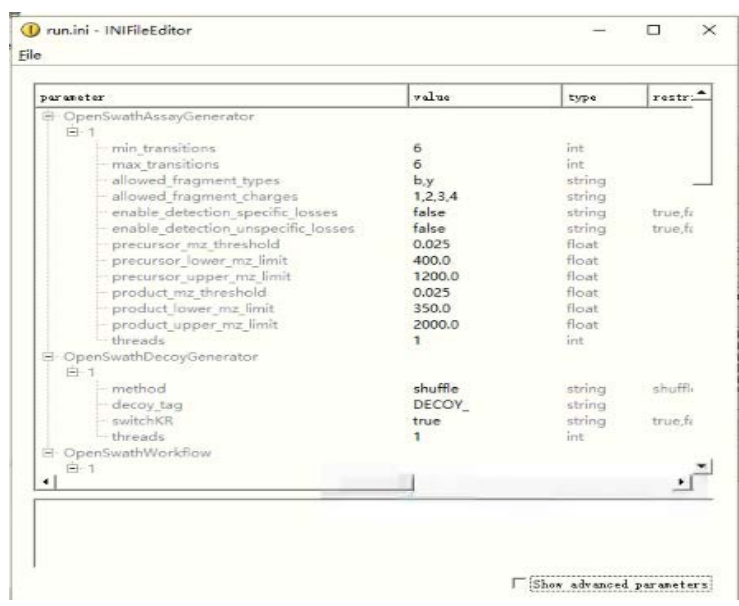

C

DIA-Umpire+QuantPipe Spectronuat_directDIA

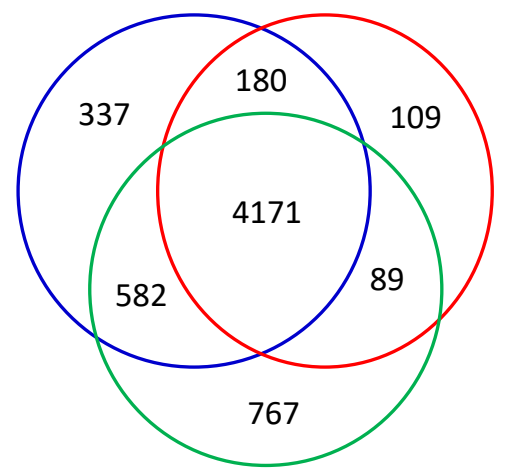

Spectronuat_library-based DIA

$E$

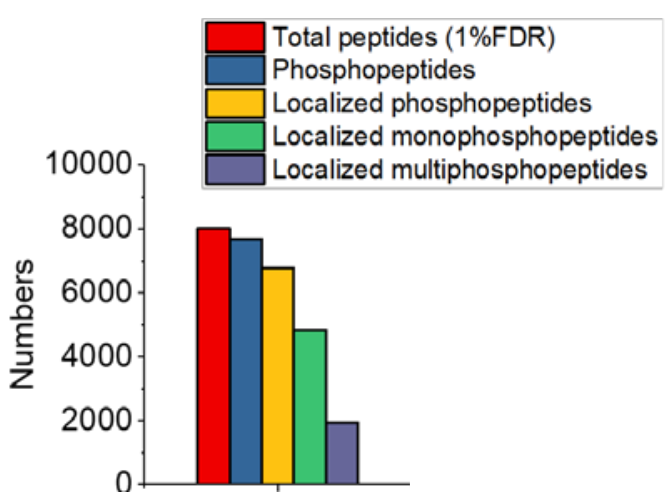

B

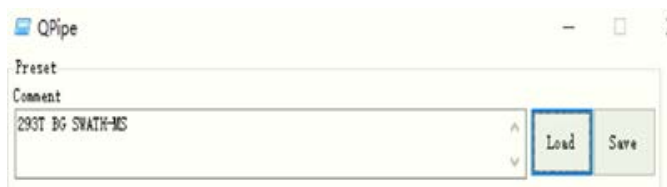

Path D: lfiles_for_QuntPipelirt. tat Villod Dilfiles_for_ouantPipelumino $\square$ IPP

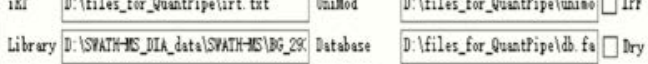

Python C: Mnacenda3! Snthindor D: Ifiles_for_OuntPipelvin_3

now/null file

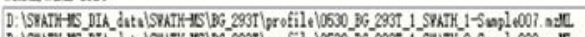

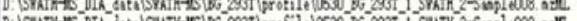

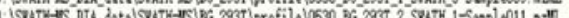

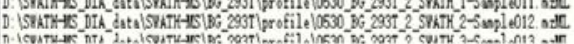

clear delete add $\square$ ose in $0.04 \div \quad \mathrm{rm}$ !

D

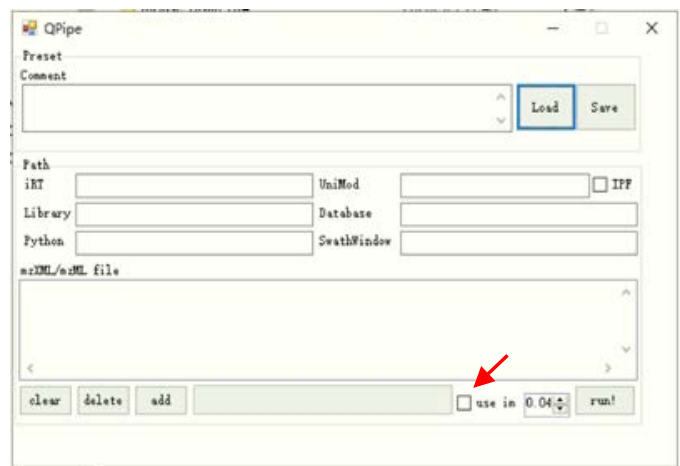

$\mathrm{F}$

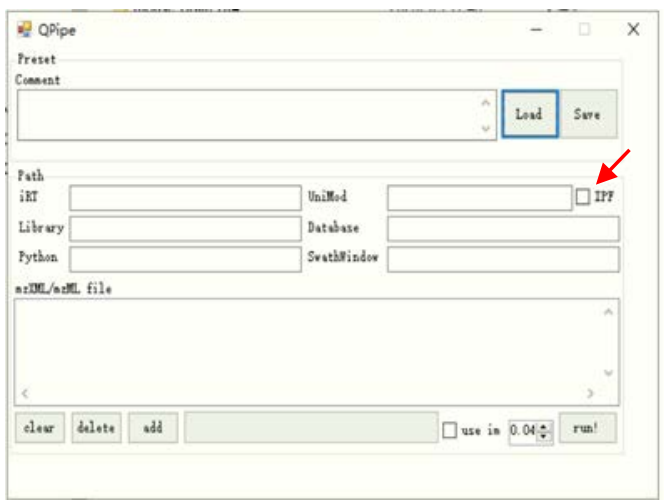

